Tropelías. Revista de Teoría de la Literatura y Literatura Comparada, 19 (2013)

Semiótica y hermenéutica del subgesto

\title{
SEMIÓTICA Y HERMENÉUTICA DEL SUBGESTO
}

\author{
José Ángel GARCÍA LANDA \\ Universidad de Zaragoza
}

parece un interesante pasaje sobre la semiótica de los subgestos en la novela
El hombre duplicado, de José Saramago (http://vanityfea.blogspot.com/
2009/07/el-hombre-duplicado.html); allí se acuña al parecer un concepto que se presta a un tratamiento más detenido ${ }^{1}$. Aquí sólo apuntaremos algunas ideas al respecto, remitiendo para una investigación ulterior y el desarrollo de una teorización más detallada, a los estudios sobre proxémica, kinésica y gestualidad de Paul Ekman, Edward T. Hall, y otros ${ }^{2}$.

El pasaje de Saramago en cuestión es un excurso del argumento, con ocasión de una reunión de profesores de instituto, tras una perorata que el protagonista Tertuliano Máximo Afonso, cargante él como Tertuliano, o como un tertuliano, les emite a sus colegas. La alocución de Tertuliano versaba sobre el estudio de la Historia, sobre «si debemos enseñarla desde detrás hacia delante o, como es mi opinión, desde delante hacia atrás» (Saramago, 2003: 58).

Se puede argumentar ciertamente que si la Historia la vemos desde el presente, quizá sería más honesto enseñarla explicítamente de esta manera, menos sujeta a la falacia narrativa. Es una cuestión relativa a la hermenéutica de la retrospección sobre la que también yo he especulado en otros escritos ${ }^{3}$. Sea como sea, Tertuliano observa los efectos que su rollo acerca de la retrospección tiene sobre los profesores. Es cita larga:

\footnotetext{
${ }^{1}$ Ver mi nota sobre El hombre duplicado (2009d) para más detalles sobre la novela. En otra nota (2009e) esbozaba algunas de las ideas que siguen.

${ }^{2}$ Puede encontrarse una bibliografía sobre comunicación no verbal en mi Bibliography of Literary Theory, Criticism and Philology (2012a).

${ }^{3}$ Ver mi artículo «Benefit of Hindsight: Polibio, Vico, Wilde y el emergentismo crítico» (2009a). O, más generalmente, los ensayos recogidos en Objects in the Rearview Mirror May Appear More Solid Than They Are (2005-2009). Apuntemos que, al margen de lo que a continuación exponemos sobre los subgestos, también tiene esta cuestión un aspecto tratable desde la hermenéutica de la retrospectividad, una dimensión que por brevedad sólo dejamos aquí apuntada: un subgesto es con frecuencia esencialmente ambiguo o ambivalente, y sólo de modo retrospectivo o retroactivo, tras un tratamiento hermenéutico, adquiere propiamente el sentido que con frecuencia se dirá que tenía en un origen. Lo mismo se aplica de hecho a cualquier modalidad de comunicación, y en especial a las más indirectas.
} 
Los efectos de la perorata fueron los de siempre, suspiros de mal resignada paciencia del director, intercambios de miradas y murmullos entre los profesores. El de Matemáticas también sonrió, pero su sonrisa fue de amistosa complicidad, como si dijera, Tienes razón, nada de esto se puede tomar en serio. El gesto que Tertuliano Máximo Afonso le envió con disimulo desde el otro lado de la mesa significaba que le agradecía el mensaje, aunque, al mismo tiempo, algo que iba adjunto y que, a falta de un término mejor, designaremos como subgesto, le recordaba que el episodio del pasillo no había sido olvidado del todo. En otras palabras, a la vez que el gesto principal se mostraba abiertamente conciliador, diciendo, Lo que pasó, pasó, el subgesto, de pie detrás, matizaba, Sí, pero no del todo. En este medio tiempo la palabra había pasado al profesor siguiente y, mientras éste, al contrario que Tertuliano Máximo Afonso, discurre con facundia, y competencia, aprovechemos para desarrollar un poco, poquísimo para lo que exigiría la complejidad de la materia, la cuestión de los subgestos, que aquí, por lo menos hasta donde llega nuestro conocimiento, se expone por primera vez. Se suele decir, por ejemplo, que Fulano, Zutano o Mengano, en una determinada situación, hicieron un gesto de esto, de eso, o de aquello, lo decimos así, simplemente, como si el esto, el eso o el aquello, duda, manifestación de apoyo o aviso de cautela, fuesen expresiones forjadas en una sola pieza, la duda, siempre metódica, el apoyo, siempre incondicional, el aviso, siempre desinteresado, cuando la verdad entera, si realmente quisiéramos conocerla, si no nos contentásemos con las letras gordas de la comunicación, reclama que estemos atentos al centelleo múltiple de los subgestos que van detrás del gesto como el polvo cósmico va detrás de la cola del cometa, porque los subgestos, para recurrir a una comparación al alcance de todas las edades y comprensiones, son como las letritas pequeñas del contrato, que cuesta trabajo descifrar, pero están ahí. Aunque resguardando la modestia que las conveniencias y el buen gusto aconsejan, nada nos sorprendería que, en un futuro muy próximo, el análisis, la identificación y la clasificación de los subgestos llegaran, cada uno por sí y conjuntamente, a convertirse en una de las más fecundas ramas de la ciencia semiológica en general. Casos más extraordinarios que éste se han visto. El profesor que hacía uso de la palabra acaba de concluir su discurso, el director va a seguir con la ronda de intervenciones, pero Tertuliano Máximo Afonso levanta enérgicamente la mano derecha, en señal de que quiere hablar. El director le preguntó si lo que tenía que comentar estaba relacionado con los puntos de vista que acababan de ser expuestos, y añadió que, en caso de ser así, las normas asamblearias en uso determinaban, como él no ignoraba, que se aguardase hasta el final de las intervenciones de todos los participantes, pero Tertuliano Máximo Afonso respondió que no señor, no es un comentario ni tiene que ver con las pertinentes consideraciones del estimado colega, que sí señor, conoce y siempre ha respetado las normas, tanto las que están en uso como las que han caído en desuso, lo que simplemente pretendía era pedir licencia para retirarse porque tenía asuntos urgentes que tratar fuera del instituto. Esta vez no fue un subgesto sino un subtono, un armónico, digamos, que vino a dar nueva fuerza a la incipiente teoría arriba expuesta sobre la importancia que deberíamos dar a las variaciones, no sólo segundas y terceras, también cuartas y quintas, de la comunicación, tanto gestual como oral. En el caso que nos interesa, por ejemplo, todos los presentes habían percibido que el subtono emitido por el director expresaba un sentimiento de alivio profundo bajo las palabras que efectivamente pronunció, Faltaría más, usted manda, a su servicio. Tertuliano Máximo Afonso se despidió con un ademán amplio de mano, un gesto para la asamblea, un subgesto para el director, y salió (Saramago, 2003: 58-60).

Hasta aquí la semiótica del subgesto según Saramago. Naturalmente esta cuestión, al margen de su posible interés para una semiología de la interacción, tiene en la novela una función estética y constructiva: podría relacionarse con esta teoría del subgesto un episodio clave de la novela: cuando Tertuliano (el hombre duplicado) le envía una barba postiza a su doble Daniel, el gesto de por sí ya es indirecto, le indica que es él, Daniel, el actor, el imitador, que Tertuliano es el «doble» original, metafísicamente más sólido, 
y que le conviene a Daniel disfrazarse para diferenciar su identidad, o para manifestarla plenamente en tanto que identidad postiza o derivada. Obsérvese que hemos tomado un ejemplo donde gesto adquiere el sentido más general de acción comunicativa no verbal. En cuanto al subgesto, podríamos decir, o el acto indirecto (acto de comunicación, aunque no de habla como los que analiza Searle en Speech Acts) es un insulto o incluso un desafío, que da lugar en el argumento de la obra a un enfrentamiento del cual saldrán los dos mal parados.

Esta cuestión de los subgestos podría relacionarse por tanto con los actos de habla indirectos de la teoría de los actos de habla, rama de la semiótica que en efecto (como sospechaba o no sospechaba Saramago) ha dado mucho que hablar, directa e indirectamente ${ }^{4}$. Una vez un significado locucionario está lingüísticamente asentado, al margen de poderse utilizar en su sentido propio, puede utilizarse de modo indirecto para transmitir sentidos ilocucionarios que no guardan correspondencia estricta con su sentido literal. Más allá de este nivel ilocucionario, también comunicativo, puede calcularse, un tanto maquiavélicamente, la producción en el oyente de efectos perlocucionarios que (dada la situación) causarán nuestras palabras. Aunque los cálculos siempre son arriesgados, y los efectos perlocucionarios, que en cualquier caso se producen, están sujetos a probabilidades e imponderables.

Pongamos, por ejemplo: yo puedo afirmar algo (sentido locucionario: afirmación) sabiendo que será captado como una amenaza por mi interlocutor (sentido ilocucionario o fuerza ilocucionaria: amenaza). Puede que lo haga con la finalidad perlocucionaria de asustar a mi interlocutor -pero quizá el efecto perlocucionario efectivo, y no buscado, sea que se ría de mí.

En suma, que un signo siempre puede servir de apoyatura para construir sobre él sentidos suplementarios en una situación dada. Hemos dicho que esta indirección se asienta sobre sentidos establecidos en el sistema lingüístico, y así es en última instancia: pero a veces el sentido «literal» es totalmente irrelevante, y la actuación comunicativa juega con niveles de indirección construidos sobre actos ya de por sí indirectos. Sobre la indirección en el lenguaje y la implicatura puede verse el capítulo 8, «Games People Play», de The Stuff of Thought de Steven Pinker ${ }^{5}$. Como señala este autor, la indirección es un aspecto crucial de la interacción comunicativa:

When people talk, they lay lines on each other, do a lot of role-playing, sidestep, shilly-shally, and engage in other forms of vagueness and innuendo. We all do this, and we expect others to do it, yet at the same time we profess to long for plain speaking, for people

\footnotetext{
${ }^{4}$ Véase, al margen de la obra de Searle ya mencionada, el libro fundacional de J. L. Austin, How to do Things with Words (1962); también entre otros Searle (1979) o Bach y Harnish (1979), y mi bibliografía «Speech Acts / Speech Act Theory» (2012c).

${ }^{5}$ Véase también la conferencia de Pinker (2010) y la versión animada de su argumentación sobre la indirección en el lenguaje (Pinker 2011). Ver mi bibliografía adicional sobre implicatura (2012d).
} 
to get to the point and say what they mean, simple as that. Such hypocrisy is a human universal. Even in the bluntest societies, people don't just blurt out what they mean but cloak their intentions in various forms of politeness, evasion and euphemism (Pinker, 2007: 374).

Lo teorizado sobre los actos de habla indirectos y otras formas de indirección en el lenguaje es aplicable en principio a otros actos comunicativos o rituales de interacción más o menos codificados: una vez establecido su sentido principal, abstracto, o de referencia, puede éste reutilizarse o reorientarse sin por ello desaparecer del todo, usándose como apoyatura o instrumento para sentidos secundarios o contextuales, para «las variaciones, no sólo segundas y terceras, también cuartas y quintas, de la comunicación, tanto gestual como oral», según lo describe Saramago. Naturalmente, este juego de señales indirectas es esencialmente ambiguo, y exceptuando los tipos más básicos y bien establecidos de indirección, no llega, por definición, a constitutir una gramática de formas semióticas construida sobre la gramática de primer nivel, sino que es en todo momento una pragmática situacional sometida a la negociación y a la interpretación -y a una potencial negativa si lo que se ha comunicado de modo indirecto fuese planteado de modo directo como una actitud o aseveración que pueda atar al sujeto. Los ejemplos de Pinker (2007) muestran claramente por qué esta ambigüedad y negabilidad son esenciales y constitutivas en la comunicación periférica, y en modo alguno son un defecto o insuficiencia comunicativa.

Y ésa podría ser una dirección posible por la que encaminar el estudio de la semiótica del subgesto. El subgesto sería, en esta aproximación teórica, un acto gestual indirecto, producido mediante un gesto que, a la vez que comunica un sentido habitualmente asociado a él, se utiliza de manera contextualmente modulada, alterado ligeramente -por ejemplo añadiéndole, o quitándole, énfasis en lo que sería su articulación habitual, o articulándolo conjuntamente con otro gesto que no suele acompañarlo. Sería un gesto que recibe una pequeña marca de algún tipo que indique al receptor que tal gesto es indirecto, que no ha de interpretarse (o no ha de interpretarse únicamente) en su sentido habitual. Quizá propiamente habría que reservar el término de «subgesto» a la alteración que recibe el gesto, pero se apreciará que puede ser complicado en la práctica (y quizá incluso en la teoría) el aislar el gesto modulado del elemento que lo modula. Mantendremos, pues, la denominación de subgesto, así entendido, para el gesto que actúa por indirección, por modulación de un sentido preestablecido, a la manera de los actos de habla indirectos -siempre teniendo en cuenta que esta cuestión de en qué medida está algo preestablecido es enormemente variable, según el grado de conocimiento mutuo que tengan los interlocutores o los códigos culturales comunes que manejen. 
Es cuestionable, sin embargo, que con esta caracterización quede suficientemente caracterizado el fenómeno descrito por Saramago. Hay aún al menos otra teoría semiótica que puede aportar algo a este estudio de los subgestos: el análisis que realiza Erving Goffman de los múltiples canales de comunicación que se superponen en una situación dada (análisis que lleva a cabo en libros como Strategic Interaction [http://garciala.blogia.com/2008/082107-teoria-paranoica-de-la-observacion-mutua.php] o Frame Analysis). Goffman distingue, por ejemplo, entre lo que se comunica intencionalmente y lo que se interpreta a partir de expresiones, Así sucede cuando dos sujetos (Harry, pongamos, y su oponente) están enzarzados en una situación de juego o competición que implica la manipulación del otro a través de un cálculo probabilístico de su actuación futura. Harry debe evaluar la situación, pero su evaluación ha de incluir al otro y sus acciones:

Podemos considerar que este segundo jugador, el otro u oponente, contribuye de dos maneras a esta evaluación. Primero, puede emitir expresiones que, cuando son recogidas por Harry, permiten a éste interpretar algo de lo que está pasando, y predecir en cierto modo lo que sucederá. (En esto el oponente, presumiblemente, no es mejor que los animales inferiores e incluso que los objetos inanimados, pues todos ellos pueden servir como fuente de información). Segundo, el oponente puede transmitir comunicaciones, es decir, trasladar declaraciones lingüísticas (o sustitutos de ellas). Éstas Harry las puede recibir (y se supone abiertamente que ha de recibirlas), y se supone que ha de ser informado por ellas (Goffman, 1970: 102, traducción mía).

El libro de Goffman Strategic Interaction está dedicado a la manera en que tanto las supuestas expresiones exudadas involuntariamente por el sujeto, como la información lingüística intencional, pueden desviarse de su sentido primario, y utilizarse para anticipar y manipular la respuesta del otro. Las señales supuestamente naturales, expresivas, son susceptibles de volverse intencionales, e incluso comunicativas a un segundo nivel, ofrecidas a la interpretación del otro con esperanza de manipularlo, haciéndole creer que tiene el dominio perspectivístico (o topsight) de la situación interaccional ${ }^{6}$. Un dominio perspectivístico que, naturalmente, se codicia para sí, y se disimula en la medida en que se tiene, para proporcionar al sujeto margen de maniobra y ases en la manga. Los signos «naturales», expresivos, pueden construirse artificialmente: las emociones pueden simularse, las supuestas huellas inconscientes, plantarse deliberadamente. Es lo que Goffman llama la degeneración de la expresión. Pero esa degeneración puede también recuperarse comunicativamente, reutilizarse, y volverse manejable como parte de la situación mutuamente entendida por los interlocutores, de modo ya sea expreso o sobreentendido. Por aquí podríamos acercarnos a otra vía para el tratamiento de la cuestión de los subgestos, que en el texto

\footnotetext{
${ }^{6}$ En «Acritical Criticism, Critical Criticism» (2010d) desarrollo más las implicaciones cognitivas e interaccionales del concepto de topsight o perspectiva dominante.
} 
de Saramago aparecen funcionando justo en la frontera donde la expresión se vuelve comunicación.

Los subgestos son analizables de la misma manera en que son analizables los cambios de tono en el discurso, o sus paréntesis, con la teoría de los marcos de Goffman. A veces ponemos entre comillas una palabra, o la subrayamos, por ejemplo para expresar ironía, «huy qué cosa tan interesante», y esto se puede hacer con cualquier marca gráfica o fónica, con la cursiva que he utilizado, o con un cambio de tono al pronunciarlo, por ejemplo estirando las comisuras de los labios. O usando ese gesto tan norteamericano-intelectual, el gesto de las comillas, levantando y moviendo dos dedos en cada mano, "así". Son señales de que un marco de referencia diferente al principal está activo; señales quizá incompletas, claro, pues requieren del oyente adecuado para que las complete: no están gramaticalizadas, sino que son esencialmente situacionales. Aunque de hecho hemos tenido que acudir en nuestra exposición, para que sean comprensibles por escrito, a ejemplos parcialmente gramaticalizados. Y aunque se pueda desarrollar, hasta cierto punto, una gramática de las situaciones, o de las transformaciones de señales, como la propuesta por Goffman.

Goffman distingue varios canales de comunicación que pueden estar activos en una situación dada. Puede haber uno principal: la situación socialmente codificada en la que diversos sujetos interactúan (por ejemplo la reunión de profesores del libro de Saramago). Pero alrededor de éste pueden surgir canales secundarios: una comunicación subordinada, «extraoficial», entre dos sujetos que comparten un contexto interactivo privado (como Tertuliano y el profesor de Matemáticas), o bien pueden darse entre los participantes diversos fenómenos de interpretación expresiva como los antes mencionados. Habrá que considerar por tanto en qué nivel se produce el subgesto: puede ser más o menos abierto para todos los interlocutores, o puede estar restringido a un canal comunicativo subordinado, accesible sólo para algunos. Considerando siempre que esta restricción intencional puede fallar, siendo el subgesto captado por un observador no previsto. O bien puede darse el caso de un (sub)gesto no intencional, que sea significativo para el intérprete, pero no haya sido emitido deliberadamente por el gesticulador. En otro lugar he expuesto cómo la teoría del sujeto de Goffman presupone una internalización de la interacción (http://garciala.blogia.com/2007/021615-goffmanel-teatro-de-la-interioridad.php): el sujeto humano y complejo se constituye mediante la división interna de roles y actitudes, interiorizando en un teatro interno de la consciencia las voces y papeles socialmente definidos ${ }^{7}$. Es interesante que, de la misma manera, los

\footnotetext{
${ }^{7}$ Ver mi artículo «Goffman: La realidad como expectativa autocumplida y el teatro de la interioridad» (2009b). Sobre Strategic Interaction, puede verse mi nota «Teoría paranoica de la observación mutua» (2008).
} 
subgestos de Saramago, que originalmente parecen un gesto subordinado a la interacción principal, pueden devenir, según una interpretación à la Goffman, un gesto que es «extraoficial» con respecto al propio gesto del sujeto, una vez este sujeto se ha convertido él mismo en una compañía teatral viviente (http://vanityfea.blogspot.com/ 2009/10/actuaciones.html) que desempeña papeles secundarios, además de los principales ${ }^{8}$.

Por aquí podríamos tender un puente entre la teoría del sujeto de Goffman y la de Freud (cuestión ambiciosa, por lo que sólo la dejaremos sugerida, a cuenta de los subgestos). El gesto que es modulado por un subgesto puede serlo de modo inconsciente para el sujeto, hemos dicho. Por esta vía, el subgesto deviene analizable en tanto que síntoma, síntoma de una tensión inconsciente para el sujeto, pero que por su existencia interfiere la acción y la manifestación conscientes del sujeto con una revelación no deseada. No es otro tipo de fenómenos el que analiza Freud en su Psicopatología de la vida cotidiana, su estudio más importante sobre actos sintomáticos:

Los actos sintomáticos, que pueden observarse en una casi inagotable abundancia tanto en los individuos sanos como en los enfermos, merecen nuestro interés por más de una razón. Para el médico constituyen inapreciables indicaciones que le marcan su orientación en circunstancias nuevas o desconocidas, y el hombre observador verá reveladas por ellos todas las cosas y a veces muchas más de las que deseaba saber. Aquel que se halle familiarizado con su interpretación se sentirá en muchas ocasiones semejante al rey Salomón, que según la leyenda oriental, comprendía el lenguaje de los animales (Freud, 1988: 881).

Tiene así este fenómeno, el subgesto entendido en tanto que síntoma, una posible dimensión de lapsus faciei, o lapsus gestus, en lugar de lapsus linguae o lapsus calami. También aquí nos las vemos con un signo emitido no intencionalmente, de hecho indeseable para la faz oficial del sujeto, por lo que es de esperar que la interpretación del mismo será conflictiva, y con frecuencia el sujeto negará su relevancia o incluso su existencia ${ }^{9}$. Resume así Freud la tesis central de su estudio sobre los actos fallidos o falsamente casuales. Su carácter es común con la neurosis, y «yace en la posibilidad de referir los fenómenos a un material psíquico incompletamente reprimido, que es

\footnotetext{
${ }^{8}$ En una serie de notas reseñadas en las referencias (2009f, g, h, i, 2010b, c) expongo y comento algunos aspectos de la teoría dramatúrgica de la acción.

9 Así lo observa Freud: «los actos inintencionados tienen que ser de continuo un manantial de malas inteligencias en el trato entre los hombres. El que los ejecuta ignora en absoluto la intención a ellos ligada, y no teniéndola, por tanto, en cuenta, no se considera responsable de los mismos. En cambio, el que los observa [los] utiliza, igual que los demás de su interlocutor, para deducir sus intenciones y propósitos, y de este modo llegar a averiguar de sus procesos psíquicos más de lo que aquél está dispuesto a comunicarle o cree haber comunicado. El adivinado se indigna cuando se le muestran tales conclusiones deducidas de sus actos sintomáticos, y las declara infundadas, puesto que al ejecutar dichos actos le ha faltado la conciencia de la intención, quejándose de mala comprensión por parte de los demás. Observada con detenimiento tal incomprensión, se ve que reposa en el hecho de comprender demasiado bien y demasiado sutilmente» (1988: 888-89).
} 
rechazado por la conciencia, pero al que no se ha despojado de toda capacidad de exteriorizarse»(1988: 931). Freud no extendió su análisis de esta psicopatología cotidiana hasta el terreno de la expresión gestual, pero se aprecia que de su teoría fácilmente se desprende que la expresión gestual puede verse afectada por el mismo tipo de alteraciones inconscientes que se da en otros tipos de expresión o de acción. Es notable, a este respecto, cómo su teoría liga lenguaje y gestualidad bajo una interpretación común, en tanto que expresiones parcialmente conscientes e intencionales, y parcialmente interferidas por asociaciones de ideas reprimidas o contradictorias:

Un estilo límpido e inequívoco demuestra que el autor está de acuerdo consigo mismo, y, en cambio, una forma de expresión forzada o retorcida nos indica la existencia de una idea no desarrollada totalmente y nos hace percibir la ahogada voz de la autocrítica del autor (1988: 819).

En cuanto al gesto facial o corporal, sus articulaciones y sus interferencias, si bien no lo trata explícitamente, se aprecia que cae fácilmente bajo la definición general de perturbaciones funcionales de la actividad motora: «Si las equivocaciones en el discurso, el cual es, sin duda alguna, una función motora, admiten una concepción como la que hemos expuesto, es de esperar que ésta pueda aplicarse a nuestras demás funciones motoras» (Freud, 1988: 857).

Los subgestos sí que han sido observados por otros, además de Saramago, y esto nos permite un breve apunte sobre su genealogía. En The First Word, Christine Kenneally remite a las investigaciones de Michael Tomasello sobre los gestos de los primates. Los gestos de los simios, dice Tomasello, caen en dos grupos principales: uno, gestos para atraer la atención; dos, los movimientos de intención. Estos últimos parecen parientes (o antepasados antropoides) de los subgestos de Saramago. «Los movimientos de intención son los principios de un movimiento efectivo, como levantar un puño para indicar una amenaza entre los humanos»(Kenneally, 2007: 124). Según Tomasello, estos gestos evolucionan entre los individuos de modo interactivo, como sigue: «yo hago de verdad una cosa, tú llegas a anticiparla, yo me fijo en tu anticipación, así que ya sólo hago el principio del movimiento» (en Kenneally, 2007: 125) -es decir, un principio de codificación de la comunicación gestual. (Y una de las raíces para el desarrollo del lenguaje [http://zaguan.unizar.es/record/3239] $)^{10}$. Estos gestos no están restringidos a los simios, claro. Los humanos, como los simios, y como otros mamíferos, hacen a veces fintas, movimientos amagados, que bastan para establecer una interacción comunicativa en un contexto dado.

\footnotetext{
${ }^{10}$ Expongo esta tesis más por extenso en «Interacción internalizada» (2007).
} 
Una dirección potencialmente provechosa para el estudio del subgesto sería la biología evolucionista. En lo que se refiere a la gestualidad en general, el propio Charles Darwin inició esta investigación en su monografía sobre la expresión de las emociones en el hombre y en los animales. La gestualidad humana, siendo mucho más elaborada por la mayor complejidad del universo comunicativo humano, puede admitir un tratamiento evolucionista, estudiando cómo unos gestos explícitos y definidos derivan de otros gestos más básicos, muchas veces a través de la interferencia gestual que cae en el ámbito de lo que hemos denominado «subgesto». Es de notar que en sus estudios de la sintomatología Freud se refiere con frecuencia a la obra de Darwin. La concepción evolucionista de la gestualidad, mediante la cual un gesto originalmente funcional se convierte en un signo meramente amagado, interpretable y manipulable, ofrece así otra vía de investigación para el estudio de la relación entre gesto y subgesto. En cuanto al gesto entendido como un fenómeno desarrollado en el seno del evolucionismo cultural, habría que acudir a las observaciones que al respecto hace Thorstein Veblen en Theory of the Leisure Class, donde la gestualidad diferenciada se convierte en una marca de posicionamiento social $^{11}$.

El subgesto, en el sentido de gesto esbozado, casi imperceptible, modulación de un gesto existente, se encuentra así en la interfaz productiva, generativa, de la creatividad gestual, y funciona en el seno de un sistema intertextual, o deberíamos decir intergestual, por referencia a gestos más establecidos o identificables frente a los cuales se delimita, o a los que reutiliza y combina.

Hay que señalar también que estos gestos y movimientos amagados son a veces intencionales y deliberados, objeto de la atención del sujeto, y a veces subconscientes, como el gesto de los babuinos nerviosos al pasarse la mano por el morro, o el de los humanos mentirosos al tocarse la nariz. Pero no porque los gestos subconscientes sean subconscientes para el sujeto dejan de ser interpretables, ya sea espontánea o reflexivamente, por los demás. De tal modo que la frontera entre lo que es subconsciente y lo que es consciente está siempre interaccionalmente debatida, y el discurso interaccional e interpretativo que recibe, interpreta y comenta la gestualidad es parte activa en su modificación y en el paso de lo subconsciente a lo consciente, y del subgesto al gesto.

Aprovechemos para introducir otra cuestión de orden interpretativo -no restringida a los subgestos, pero que les atañe en tanto que fenómeno sujeto a interpretación y con frecuencia a conflicto de interpretaciones. Sucede en este caso

\footnotetext{
${ }^{11}$ Sobre Darwin y Veblen, ver el artículo de la Wikipedia dedicado al libro de Darwin The Expression of the Emotions in Man and Animals. Sobre el uso que se hace de la proxémica y la gestualidad en su representación literaria, es muy interesante el libro de Fernando Poyatos La comunicación no verbal (1994). Para más estudios sobre gestualidad, véase mi bibliografía sobre gestos (2012b).
} 
como sucede con otros fenómenos de significado debatible -con los sueños, pongamos, o más generalmente con la interpretación de las expresiones (literarias, artísticas, etc.) de significado ambiguo o contestable. El significado de un gesto (de un sueño, etc.) no está en el gesto mismo, sino en el tratamiento interpretativo que se le da. Un tratamiento que por supuesto se enraíza con frecuencia en una noción de lo que significa el gesto (sueño, poema, etc.) «en sí», pero que por su apertura al debate o a la elaboración interpretativa, supone una expansión, un asentamiento y una reutilización del significado. Así pues, el «auténtico» significado de un sueño (gesto, etc.) puede ser indeterminable, pero con frecuencia es determinable cómo se ha interpretado en una situación dada, qué sentido se le ha asignado, en el marco de una interacción interpretativa o de un protocolo de asignación de significados. Aquí nos remitimos a la teoría del significado expuesta por los interaccionalistas simbólicos como G. H. Mead o Herbert Blumer, que sitúan el significado de un fenómeno no en el fenómeno en sí, ni en la mente subjetiva del intérprete, sino en un proceso de interacción social que asigna sentidos y los reutiliza ${ }^{12}$. Es en este sentido en el que muchas veces podemos estar razonablemente seguros del significado de un subgesto: cuando nos referimos al significado que se la ha asignado en un proceso de interpretación, o en un contexto discursivo determinado en el que los interlocutores llegan a un acuerdo sobre el sentido de tal gesto.

Pero volvamos a lo que es intrínsecamente subgestual. Un subgesto presupone, como señala Saramago, el gesto principal al que se añade o al que se refiere. Por tanto es una variación o modulación del gesto primario o principal, variación que le imprime una dirección o distorsión determinada. Esto quiere decir que los subgestos, aparte de ir subordinados a «otro» gesto que es el principal, pueden considerarse a su vez formas derivadas, o atenuaciones, de un tercer gesto -otro gesto principal, o mejor original, que no es realizado sino de forma transformada, a través del subgesto. Por ejemplo, en lugar de tocarnos la nariz tras mentir, podríamos sólo amagar este gesto. En el ejemplo de Saramago, el gesto principal que se articula y al que se adhiere el subgesto es un gesto de conciliación o de solidaridad hacia el profesor de Matemáticas, pero el gesto principal que no se articula y del cual deriva el subgesto es un gesto de confrontación o de resentimiento $-\mathrm{y}$ no es articulado abiertamente como tal, sino sólo en su forma modulada o derivada como subgesto. Esta modulación será más interpretable en tanto que tal (esto es, podrá afinarse más la interpretación) entre quienes conozcan las características gestuales e interaccionales del sujeto gesticulante. Es decir, los subgestos, como sucede con otros matices de la comunicación indirecta o de la

\footnotetext{
${ }^{12}$ Ver Blumer, Symbolic Interactionism (1986) y mi artículo «Pragmática, interaccionismo, y análisis crítico del discurso» (2005).
} 
interpretación elaborada, encuentran su lugar más propio entre sujetos que guardan entre sí una estrecha familiaridad, que son capaces de captar los matices de los signos que se dirigen o que emiten, o capaces de modular esos matices en base a interacciones anteriores y a gramáticas locales. Gramáticas por así llamarlas, pues son codificaciones provisionales y variables, a la par que personalizadas, o idiosincráticas, desarrolladas por sujetos que se tienen estudiados, y han desarrollado una larga familiaridad entre sí, o consigo mismos. «Everything is a cipher»-es una de las apoyaturas de aquel relato de Nabokov sobre un viejo matrimonio, «Signs and Symbols» ${ }^{13}$ (http://garciala.blogia.com/2007/052202-out-of-character.php): «In silence he sat down on the steps and in silence rose when some ten minutes later she came, heavily trudging upstairs, wanly smiling, shaking her head in deprecation for her silliness» (Nabokov, 1960: 55). En esta escena, la esposa ha dejado al marido encerrado fuera de casa por olvido; el amago de sonrisa y el gesto de negación son una combinación gestual que le comunica a sí misma, y al marido, su autoevaluación. En la ausencia de palabras, observamos la reducción semiótica por la cual opera la interacción comunicativa: el gesto reducido sustituye al gesto pleno, o a la expresión articulada, cuando es contextualmente suficiente para la interpretación ${ }^{14}$.

Son grandes lectores de signos y símbolos, y de subgestos, los viejos matrimonios. Pero lo mismo puede aplicarse a cualquier sociedad parcialmente cerrada o cualquier comunidad interpretativa bien trabada, que deriva su identidad, en parte, de la capacidad de leer ciertos signos que para el profano son ilegibles, o insignificantes, o inexistentes.

\section{Referencias}

AUSTIN, J. L. (1962): How to Do Things with Words. Ed. J. O. Urmson. Oxford, Clarendon Press.

BACH, K., y R. M. HARNISH. (1979): Linguistic Communication and Speech Acts. Cambridge (MA), MIT Press.

BLUMER, H. (1986): «The Methodological Position of Symbolic Interactionism», en Blumer, Symbolic Interactionism. Berkeley, University of California Press, pp. 160.

\footnotetext{
${ }^{13}$ «Signs and Symbols» es uno de los ejemplos que comento en «Out of Character: Narratología del sujeto y su trayectoria vital» (2010a).

${ }_{14}$ Añádase, por completar el mapa de la comunicación subliminal, que este episodio es una variante «envejecida y triste» de un episodio amoroso juvenil que cierra la novela autobiográfica de Nabokov Dar (The Gift). Los protagonistas de ambos relatos son trasuntos del propio Nabokov y su esposa, y la interpretación plenamente desengañada de la escena de las llaves, que aquí en «Signs and Symbols» es una especie de subgesto textual, presupone el conocimiento de la articulación original de ese gesto en Dar. También la sub-gestualidad textual se basa, como se ve, en una intertextualidad.
} 
DARWIN, C. (1872): The Expression of the Emotions in Man and Animals. En The Complete Work of Charles Darwin Online, en http://darwin-online.org.uk/ content/frameset?itemID=F1142\&viewtype=text\&pageseq=1 (última consulta, 103-2012).

FREUD, S. (1988): Psicopatología de la vida cotidiana. Trad. Luis López Ballesteros y de Torres. En Freud, Obras completas. Madrid, Orbis, vol. 4, pp. 755-931.

GARCIA LANDA, J. A. (2005): «Pragmática, interaccionismo, y análisis crítico del discurso». Vanity Fea 21 nov., en http://garciala.blogia.com/2005/112201pragmatica-interaccionismo-y-analisis-critico-del-discurso.php (última consulta, 10-3-2012).

(2005-9): Objects in the Rearview Mirror May Appear More Solid Than They Are: Retrospective / Retroactive Narrative Dynamics in Criticism. Universidad de Zaragoza, en http://www.unizar.es/departamentos/filologia_inglesa/garciala/ publicaciones/retroretro.html (última consulta, 10-3-2012).

(2007): «Internalized Interaction: The Specular Development of Language and the Symbolic Order / Interacción internalizada: el desarrollo especular del lenguaje y el orden simbólico». Social Science Research Network (diciembre), en http://ssrn.com/abstract=1073782 (última consulta, 10-3-2012).

(2008): «Teoría paranoica de la observación mutua". Vanity Fea 21 agosto, en http://garciala.blogia.com/2008/082107-teoria-paranoica-de-la-observacionmutua.php (última consulta, 10-3-2012).

(2009a): «Benefit of Hindsight: Polibio, Vico, Wilde y el emergentismo crítico». Zaguán 24 marzo 2009, en http://zaguan.unizar.es/record/3215 (última consulta, 10-3-2012).

(2009b): «Goffman: La realidad como expectativa autocumplida y el teatro de la interioridad». Zaguán 29 mayo 2009, en http://zaguan.unizar.es/record/3358 (última consulta, 10-3-2012).

(2009c): «Out of Character: Narratología del sujeto y su trayectoria vital». Zaguán 18 junio 2009, en http://zaguan.unizar.es/record/3373 (última consulta, 10-3-2012).

(2009d): «El hombre duplicado». Vanity Fea 11 julio, en http://vanityfea.blogspot.com/2009/07/el-hombre-duplicado.html (última consulta, 10-3-2012).

(2009e): «Semiótica del subgesto». Vanity Fea 12 julio, en http://vanityfea.blogspot.com/2009/07/semiotica-del-subgesto.html (última consulta, 10-3-2012). 
Tropelías. Revista de Teoría de la Literatura y Literatura Comparada, 19 (2013)

Semiótica y hermenéutica del subgesto

- (2009f): «Actuaciones». Vanity Fea 21 octubre, en http://vanityfea.blogspot.com/2009/10/actuaciones.html (última consulta, 10-32012).

(2009g): «Equipos y sujetos (... al equipo) ». Vanity Fea 14 noviembre, en http://vanityfea.blogspot.com/2009/11/equipos-y-sujetos-al-equipo.html (última consulta, 10-3-2012).

(2009h): «Redes, regiones y públicos». Vanity Fea 21 diciembre, en http://vanityfea.blogspot.com/2009/12/redes-y-regiones.html (última consulta, 103-2012).

(2009i): «El interlocutor interiorizado». Vanity Fea 29 diciembre, en http://vanityfea.blogspot.com/2009/12/el-interlocutor-interiorizado.html (última consulta, 10-3-2012).

(2010a): «Out of Character: Narratología del sujeto y su trayectoria vital». En Los caminos de la lengua: Estudios en homenaje a Enrique Alcaraz Varó. Ed. J. L. Cifuentes et al. San Vicente del Raspeig (Alicante): Publicaciones de la Universidad de Alicante, pp. 1053-63.

- (2010b): «Double Talk». Vanity Fea 23 enero, en http://vanityfea.blogspot.com/2010/01/double-talk.html (última consulta, 10-32012).

(2010c): «Versiones de la realidad». Vanity Fea 24 enero, en http://vanityfea.blogspot.com/2010/01/versiones-de-la-realidad.html (última consulta, 10-3-2012).

(2010d): «Acritical Criticism, Critical Criticism: Critical Interaction, Reframing and Topsight». En Con/Texts of Persuasion. Ed. Beatriz Penas et al. Kassel: Edition Reichenberger, 2011, pp. 233-68. Una versión en español: "Crítica acrítica, crítica crítica". Zaguán 4 abril, en http://zaguan.unizar.es/record/3228 (última consulta, 10-3-2012).

(2012a): «Proxemics, Kinesics and Nonverbal Communication». A Bibliography of Literary Theory, Criticism, and Philology, $17^{\mathrm{a}}$ ed., en http://www.unizar.es/departamentos/filologia_inglesa/garciala/bibliography/Subje cts/7.Semiotics/Proxemics, Kinesics.doc (última consulta, 10-3-2012).

(2012b): «Gestures». En A Bibliography of Literary Theory, Criticism, and Philology. $17^{\mathrm{a}}$ ed., en http://www.unizar.es/departamentos/filologia_inglesa/ garciala/bibliography/Subjects/z.Other.subjects/Motifs/G/Gestures.doc (última consulta, 10-3-2012).

(2012c): «Speech Acts / Speech Act Theory». En A Bibliography of Literary Theory, Criticism, and Philology. $17^{\mathrm{a}}$ ed., en http://www.unizar.es/ 
departamentos/filologia_inglesa/garciala/bibliography/Subjects/6.Linguistics/3.Li nguistic.categories/P- Z.linguistic.categories/Pragmatics/Speech.acts.doc (última consulta, 10-3-2012).

(2012d): «Implicature». A Bibliography of Literary Theory, Criticism, and Philology. $17^{\mathrm{a}}$ ed., en http://www.unizar.es/departamentos/filologia_inglesa/ garciala/bibliography/Subjects/6.Linguistics/3.Linguistic.categories/P-Z.linguistic. categories/Pragmatics/Implicature.doc (última consulta, 10-3-2012).

GOFFMAN, E. (1970): Strategic Interaction. (Conduct and Communication, 1). Filadelfia, U of Pennsylvania P.

(1986): Frame Analysis: An Essay on the Organization of Experience. Boston, Northeastern University Press.

KENNEALLY, C. (2007): The First Word: The Search for the Origins of Language. Londres, Penguin.

NABOKOV, V. (1960): «Signs and Symbols», en Nabokov's Dozen. Harmondsworth, Penguin, pp. 67-74.

(1979): Five Novels [Lolita, The Gift, Invitation to a Beheading, King Queen Knave, Glory]. Londres, Collins.

PINKER, S. (2007): The Stuff of Thought: Language as a Window into Human Nature. Nueva York, Viking.

- (2010): «The Stuff of Thought: Language as a Window into Human Nature». YouTube-RSA 4 feb., en http://youtube/5S1d3cNge24 (última consulta, 10-32012).

(2011): «RSA Animate: Language as a Window into Human Nature». Vídeo en red. YouTube-RSA 10 feb., en http://www.youtube.com/watch?feature= player_embedded\&v=3-son3EJTrU (última consulta, 10-3-2012).

POYATOS, F. (1994): La comunicación no verbal. I. Cultura, lenguaje y comunicación. II. Paralenguaje, kinésica e interacción. III. Nuevas perspectivas en novela y teatro y en su traducción. (Biblioteca Española de Lingüística y Filología). Madrid, Istmo.

SARAMAGO, J. (2002): O Homem Duplicado. Lisboa, Caminho. (2003): El hombre duplicado. Trad. Pilar del Río. Madrid: Santillana-Alfaguara.

SEARLE, J. R. (1969): Speech Acts: An Essay in the Philosophy of Language. Cambridge, Cambridge UP.

(1979): Expression and Meaning: Studies in the Theory of Speech Acts. Cambridge, Cambridge UP.

VEBLEN, T. (1973): The Theory of the Leisure Class. Boston, Hougton Mifflin. 
Tropelías. Revista de Teoría de la Literatura y Literatura Comparada, 19 (2013)

Semiótica y hermenéutica del subgesto

WIKIPEDIA (2012): «The Expression of the Emotions in Man and Animals». Wikipedia: The Free Encyclopedia, en http://en.wikipedia.org/wiki/ The_Expression_of_the_Emotions_in_Man_and_Animals (última consulta, 10-32012). 